\title{
Article
}

\section{Multinational Experiences in Reducing and Preventing the Use of Restraint and Seclusion}

LeBel, Janice L., Duxbury, Joy, Putkonen, Anu, Sprague, Titia, Rae, Carolyn and Sharpe, Joanne

Available at http://clok.uclan.ac.uk/13755/

LeBel, Janice L., Duxbury, Joy ORCID: 0000-0002-1772-6874, Putkonen, Anu, Sprague, Titia, Rae, Carolyn and Sharpe, Joanne (2014) Multinational

Experiences in Reducing and Preventing the Use of Restraint and Seclusion. Journal of Psychosocial Nursing and Mental Health Services, 52 (11). pp. 2229. ISSN 0279-3695

It is advisable to refer to the publisher's version if you intend to cite from the work. http://dx.doi.org/10.3928/02793695-20140915-01

For more information about UCLan's research in this area go to

http://www.uclan.ac.uk/researchgroups/ and search for <name of research Group>.

For information about Research generally at UCLan please go to http://www.uclan.ac.uk/research/

All outputs in CLoK are protected by Intellectual Property Rights law, including Copyright law. Copyright, IPR and Moral Rights for the works on this site are retained by the individual authors and/or other copyright owners. Terms and conditions for use of this material are defined in the policies page.

\section{CLoK}

Central Lancashire online Knowledge www.clok.uclan.ac.uk

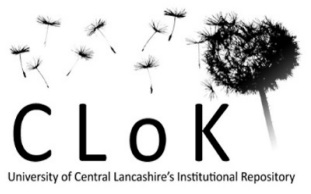




\title{
Multinational Experiences in Reducing and Preventing the Use of Restraint and Seclusion
}

\author{
Janice L. LeBel, PhD, ABPP; Joy A. Duxbury, PhD, MA, BSc, RMN; Anu Putkonen, MD, PhD; Titia \\ Sprague, MBBS, FRANZCP; Carolyn Rae, RGN, RSCN, RMN; and Joanne Sharpe, BAppSc(OT)
}

\begin{abstract}
Restraint and seclusion (R/S) have been used in many countries and across service sectors for centuries. With the recent and increasing recognition of the harm associated with these procedures, efforts have been made to reduce and prevent $\mathrm{R} / \mathrm{S}$. Following a scathing media exposé in 1998 and congressional scrutiny, the United States began a national effort to reduce and prevent $\mathrm{R} / \mathrm{S}$ use. With federal impetus and funding, an evidence-based practice, the Six
\end{abstract}

Core Strategies ${ }^{\circledR}$ to Prevent Conflict, Violence and the Use of Seclusion and Restraint, was developed. This model was widely and successfully implemented in a number of U.S. states and is being adopted by other countries, including Finland, Australia, and the United Kingdom. Recently, the first cluster randomized controlled study of the Six Core Strategies in Finland provided the first evidence-based data of the safety and effectiveness of a coercion prevention methodology. Preliminary findings of some of the international efforts are discussed in the current article. Reduction in R/S use and other positive outcomes are also reported. [Journal of Psychosocial Nursing and Mental Health Services, $x x(\mathrm{x}), \mathrm{xx}-\mathrm{xx}$.]

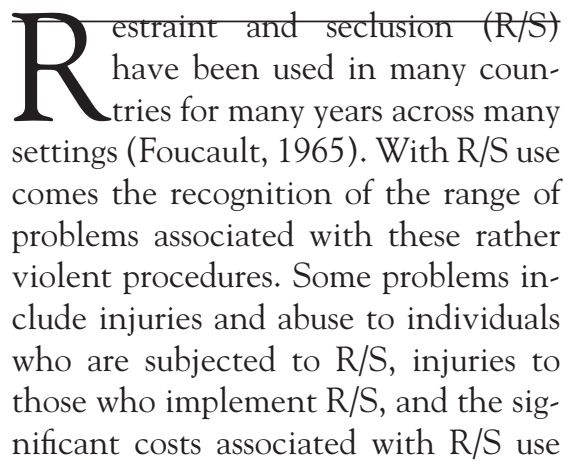


(LeBel, 2011). Along with this knowledge comes remedies to these problems; remedies vary but often take the form of implementing measures to reduce use and prevent harm (LeBel, 2008).

Currently, leaders from several countries, serving different populations, are reexamining their use of R/S and implementing the same evidence-based model, which includes a preventionoriented organizational approach to reducing conflict, violence, and the factors that contribute to these coercive methods. These leaders predominantly represent public sector mental health inpatient services in countries such as the United States, Australia, Finland, the United Kingdom, Canada, and New Zealand. Although the specific impetus to pursue this model varies, the intent to minimize or avoid harm to all involved in R/S events is a common motivation for each country and setting. International efforts to reduce $\mathrm{R} / \mathrm{S}$ have not been well discussed, and this work is important. The leaders who collaborated on the current article were chosen based on their work and ability to participate in the timeframes provided in regard to publication of the current article.

The model that is being used by facilities in Finland, Australia, and the United Kingdom is based on and referred to as the Six Core Strategies ${ }^{\odot}$. This evidence-based best practice approach was developed in the United States following scathing national media reports (Weiss, Altimari, Blint, \& Megan, 1998) and congressional investigations, which documented R/S deaths and abuses more than 15 years ago (Huckshorn, 2006; Huckshorn \& LeBel, 2009). National scrutiny resulted, and millions of federal dollars were appropriated and distributed by the Substance Abuse Mental Health Services Administration (SAMHSA) to develop (a) alternatives to the use of R/S and (b) tools to reduce the use of these procedures with the ultimate goal of R/S elimination (Huckshorn \& LeBel, 2009).

\section{THE SIX CORE STRATEGIES}

In 2002, the National Association of State Mental Health Program Directors' (NASMHPD) Office of Technical Assistance received funding from SAMHSA to develop a curriculum to address the problem of $\mathrm{R} / \mathrm{S}$ use in inpatient settings, which typically resulted from conflict, coercion, and violence in the care setting. NASMHPD staff conducted a thorough review of the literature, met with individuals with lived experience of $\mathrm{R} / \mathrm{S}$, and convened multiple working sessions with national experts-all of whom had successfully reduced R/S in their work settings and sustained the reduction for several years (Huckshorn, 2006; Huckshorn \& LeBel, 2009; NASMHPD, 2014). The experts identified similar themes and methods used in their respective reduction efforts, which formed the basis of the Six Core Strategies (Huckshorn, 2006; Huckshorn \& LeBel, 2009; NASMHPD, 2014).

The Six Core Strategies model was articulated and imbedded in a prevention-oriented, trauma-informed care framework that approached the problem of restrictive procedure use from a quality improvement perspective. The specific core strategies are (a) active leadership toward organizational change; (b) using data to inform practice; (c) developing the workforce; (d) using R/S prevention tools; (e) actively including consumers and advocates in the care setting; and (f) rigorously debriefing $\mathrm{R} / \mathrm{S}$ events after they occur (Huckshorn, 2006; Huckshorn \& LeBel, 2009; NASMHPD, 2014).

Each strategy was developed into a didactic presentation using current literature, practice-based evidence, and pragmatic examples of specific implementation tasks associated with each strategy. In addition, an action planning template and implementation checklist were developed to accompany the curriculum (NASMHPD, 2014). Together, these tools support the development of an R/S reduction/ prevention action plan and provide organizations with specific tools to assess their change efforts against multiple activities over time (Human Services
Research Institute, 2009; NASMHPD, 2014).

\section{IMPLEMENTATION IN THE UNITED STATES}

\section{Background and Process}

As part of the initial development and dissemination of the Six Core Strategies curriculum, SAMHSA awarded eight U.S. states 3-year incentive grants to implement the model and study the effect of the approach on $\mathrm{R} / \mathrm{S}$ use in the participating state facilities. The results from the research cohort demonstrated significant positive results with facilities that had fully implemented the strategies, with (a) more than $70 \%$ reduction in hours of seclusion use and percentage of consumers who experienced seclusion and (b) more than $50 \%$ reduction in hours of restraint use and percentage of consumers who experienced restraint in care (NASMHPD, 2014). The positive results of the first cohort established a strong base of evidence to warrant formal inclusion in the National Registry of Evidence-Based Programs and Practices (2012). Results from the second cohort are pending and have yet to be reported.

Since its development, the Six Core Strategies have been widely taught throughout the United States (LeBel, 2011). NASMHPD's faculty have provided training to more than 6,000 mental health leaders and staff from public and private sector organizations, who represent 47 states and several U.S. territories. Several other countries have requested and received this training, including Canada, Australia, New Zealand, Germany, Turkey, Sweden, Finland, the United Kingdom, and the Czech Republic, and have begun or are planning to implement the model. Preliminary findings from some of these efforts are now being reported.

\section{Outcomes}

After codification and implementation of the Six Core Strategies in many different settings across the United States, success stories were reported, as well as other practice advance- 
ments and pragmatic alternatives to R/S use (LeBel, 2011). Examples of significant $\mathrm{R} / \mathrm{S}$ reductions occurred in: (a) Massachusetts, where two continuing care hospitals reduced their use of $\mathrm{R} / \mathrm{S}$ by more than $90 \%$ in less than 3 years (Huckshorn, 2012; LeBel, 2011; NASMHPD, 2014); (b) Maryland, where university hospital staff participated in Six Core Strategies training in 2005 and reduced their use of R/S by $75 \%$ in the same year, with no increase occurring in injuries to staff or consumers (Lewis, Taylor, \& Parks, 2009); and (c) Pennsylvania, where Barton, Johnson, and Price (2009) eliminated R/S use in their facility, after attending the same Six Core Strategies training as Maryland hospital staff, and have not used these procedures for more than 4 years. Barton et al. (2009) also noted a $22 \%$ decrease in medication use.

Likewise, a child and adolescent psychiatric facility in Minnesota conducted a 33-month retrospective review of R/S use before and after Six Core Strategies training and reported more than 66\% reduced use and 50\% fewer youth experiencing $\mathrm{R} / \mathrm{S}$ at their hospital (Azeem, Aujla, Rammerth, Binsfeld, \& Jones, 2011). The medical leadership at this facility took this approach to a hospital in Connecticut and also achieved significant restraint reduction $(86 \%)$ and seclusion reduction (40\%) within the year (LeBel, Huckshorn, \& Caldwell, 2014). At a southern Florida university hospital, R/S was discontinued after the hospital's second anniversary of being R/S-free; however, due to forensic admissions, this facility has returned to using $\mathrm{R} / \mathrm{S}$, but at low rates (NASMHPD, 2014).

In Illinois, two psychiatrists in one large hospital serving forensic and civilly committed patients reduced R/S use by $95 \%$ within 1 year and promoted the use of the Six Core Strategies (Hardy \& Patel, 2011). In New York City, the Health and Hospital Corporation trained more than 760 leadership and direct care staff in the Six Core Strategies and implemented the framework in their 11 facilities with a combined capacity of more than 1,117 beds; they re- alized not only $\mathrm{R} / \mathrm{S}$ reduction, but also a reduction in duration and injuries to consumers served in their psychiatric emergency services (Wale, Belkin, \& Moon, 2011).

In addition to $\mathrm{R} / \mathrm{S}$ reductions and, in some cases, elimination, other benefits resulted from this or similar approaches that informed the Six Core Strategies development (LeBel, Huckshorn, \& Caldwell, 2010). Several organizations reported significant reductions in staff turnover and related costs, as well as decreased staff injuries, absenteeism, hiring, and retraining costs (LeBel, 2011; LeBel \& Goldstein, 2005). Other agencies reported that Six Core Strategies implementation resulted in decreased workers' compensation premiums, claims, and medical costs, as well as increased staff satisfaction and retention (LeBel, 2011). Benefits to consumers (i.e., decreased injuries, lengths of stay, medications, and incidents of rehospitalization, and increased treatment satisfaction) were identified in successful R/S projects (LeBel, 2011).

Aside from facility-specific R/S reductions, several states embarked on large-scale R/S reduction efforts and reported significant changes in practice (LeBel, 2011). Innovations and practical alternatives to $\mathrm{R} / \mathrm{S}$ were implemented (e.g., sensory interventions and methods) (LeBel \& Champagne, 2010). Key catalysts to service transformation and culture change were also identified through this process (LeBel \& Urff, in press). Key catalysts include trauma-informed care and full consumer inclusion. The catalysts are not mutually exclusive or functional tasks, but rather they are integrally related conceptual positions that require a concerted philosophical shift toward person-centered practice (Jennings, 2007; NASMHPD, 2014).

\section{IMPLEMENTATION IN FINLAND \\ Background}

Finland is a large, socially and ethnically homogenic, Nordic country of 5.4 million individuals. The counties organize and fund psychiatric inpatient services. R/S are allowed only during involuntary hospital care. The Finnish National Board of Health and Welfare and the local state authorities obtain the R/S data. The highest $\mathrm{R} / \mathrm{S}$ rates have been recorded in the two state hospitals. The larger of the two hospitals includes a department of forensic psychiatry from a local university. In addition to inpatient services, the hospital also provides forensic psychiatric examinations, education, and research. During the randomized controlled trial to implement the Six Core Strategies, more than one half of the inpatients had forensic involvement, and $45 \%$ had demonstrated behavior that was considered too dangerous or too difficult to be treated in local hospitals. Almost all patients were Finnish-speaking Caucasians who had psychotic disorders with violent behavior and were in involuntary care; $86 \%$ were men. The hospital comprised 13 wards (286 beds) for adults and one ward with 20 beds for adolescents. The hospital employed approximately 800 individuals in 604 positions.

The coercion reduction project and the randomized controlled study of the Six Core Strategies took place during 2008 (information year) and 2009 (intervention year). This work was funded by the National Board for Health and Welfare to reduce the high $\mathrm{R} / \mathrm{S}$ rates and determine if it was possible to reduce R/S without a resulting increase in violence. Controlled data on the safety and effectiveness of coercion reduction strategies had not been published, and the routine use of $\mathrm{R} / \mathrm{S}$ was postulated as related to safety. By using control wards, it was also possible to eliminate the effect of the confounding factors on $\mathrm{R} / \mathrm{S}$ and violence (e.g., organizational and seasonal factors). The academic task force (i.e., the team) included a senior nurse, a cultural anthropologistpsychotherapist counselor, and a forensic psychiatrist-researcher who lead the project, planned the intervention; tailored the tools; and educated, supervised, and helped the wards implement the Six Core Strategies (Putkonen et al., 2013). 


\section{Process}

Leadership Toward Organizational Change. During 2008, the entire hospital was informed of the R/S prevention effort. The team visited five U.S. state hospitals in Pennsylvania and Florida to observe the Six Core Strategies in practice and was trained by leaders and experts in coercion reduction. The team also informed the hospital leaders who worked in the steering group of the work being done in the United States. In 2009, the team supervised staff and patients of the two intervention wards for a period of 6 months and then supported the use of the Six Core Strategies during the subsequent 6 months. Staff, patients, and doctors critically reviewed problems, rules, and practices together. Staff received weekly information on crisis prevention tools and the risks and trauma associated with R/S use. They were also given assistance in developing individualized prevention strategies and alternatives to seclusion.

Consumer and Family Involvement. In consumer specialist meetings, the service users discussed their personal experiences with violence and coercion, triggers of violence, and effective calming activities. They also suggested new ways and practices to decrease fear, violence, and coercion and brainstormed with staff and doctors about the ward rules and practices in community meetings. Some patients and staff improved the courtyard to enhance the environment of care, and others wrote, photographed, and illustrated a book, titled Behind Locked Doors (hospital publication only).

Using Data to Inform Practice. Staff discussed the monthly coercion and violence data with the senior nurse and leaders in the monthly steering group meetings. Data were graphed, posted, and widely shared with staff on a monthly basis. Individual graphics of violence and seclusion were used in counseling and crisis planning, and a progress sheet was created and distributed to encourage staff to continue to reduce $\mathrm{R} / \mathrm{S}$.

Restraint and Seclusion Prevention
Tools. Crisis prevention tools helped with individual crisis prevention, deescalation of tense situations, and coping with crises. These tools included a questionnaire of traumatic experiences and violent behavior, triggers, warning signs, calming activities, and daily activities. The individual crisis plan (i.e., an agreement on the calming activities to be used if the warning signs of anger appeared) was revised after each crisis. Each morning the project's senior nurse and cultural anthropologistpsychotherapist counselor met with staff to discuss the violent incidents that occurred within the past day and the practices, restrictions, and alternative methods used according to the post-event analysis sheet. These meetings identified and praised successful interventions and helped staff improve their practices.

\section{Outcomes}

The differences of the monthly R/S use between intervention wards versus control wards were significant for both coercion days (i.e., the proportion of patient days when any $\mathrm{R} / \mathrm{S}$ or observation was used $[p=0.001]$ and for $\mathrm{R} / \mathrm{S}$ time $[p=0.001])$, but not for violence, which decreased in both groups $(p=0.91)$ after the information of the strategies was provided for all wards. The monthly proportion of patient days with any coercion declined from $30 \%$ to $15 \%$ for intervention wards (incidence rate ratio $[\mathrm{IRR}]=0.88 ; 95 \%$ confidence interval $[\mathrm{CI}]=0.86,0.90 ; p<0.001$ ) versus $25 \%$ to $19 \%$ for control wards $(\mathrm{IRR}=0.97 ; 95 \% \mathrm{CI}=0.93,1.01$; $p=0.056)$. R/S time decreased from 110 to 56 hours per 100 patient days for intervention wards $($ IRR $=0.85$; $95 \% \mathrm{CI}=0.78,0.92 ; p<0.001)$ but increased from 133 to 150 hours for control wards $(\mathrm{IRR}=1.09 ; 95 \% \mathrm{CI}=0.94$, $1.25 ; p=0.24)$. Incidence of violence decreased from $1.1 \%$ to $0.4 \%$ for the intervention wards and from $0.1 \%$ to $0 \%$ for control wards. The IRR for annual $\mathrm{R} / \mathrm{S}$ time (per patient time) in the entire hospital declined steadily from the beginning of the information year. The IRR for annual R/S time, compared with 2007, was $0.75(95 \% \mathrm{CI}=0.73$, $0.78)$ in 2008 and 0.49 (95\% CI $=0.47$, 0.51 ) in 2009. Reporting of patient-tostaff violence increased from 13 reports (2007) to 18 (2008) and 22 (2009) reports. However, patient-associated sick days decreased approximately $70 \%$, and the mean duration of a patient-associated sick leave shortened approximately $81 \%$ during the project.

The study demonstrated that it is possible to significantly reduce $\mathrm{R} / \mathrm{S}$ use with the Six Core Strategies without an increase in violence. Because this reduction was achieved in the care of a stratified, nationally representative sample of individuals with schizophrenia who display severe or chronic violent behavior, similar or even greater reduction may be possible under less extreme circumstances (Putkonen et al., 2013).

\section{Current Status and Next Steps}

In 2010, the Finnish National Board for Health and Welfare asked representatives from each psychiatric hospital to participate in a national task force for $\mathrm{R} / \mathrm{S}$ reduction. They met regularly in Helsinki over a 3-year period and participated in a formal 2-day training at the pilot hospital on the use of the Six Core Strategies in 2011; approximately 300 other psychiatric experts from different parts of the country were in attendance. The task force created a book and a toolkit of coercion reduction practices, which is now being edited by the team leaders. The aim was national $\mathrm{R} / \mathrm{S}$ reduction and the creation of an $\mathrm{R} / \mathrm{S}$ reduction plan for each psychiatric hospital. Many hospitals initiated their own R/S reduction projects and reduced their use with the Six Core Strategies. However, the inter-facility comparisons are problematic. The task force members suggested that a national coercion reduction in Finland necessitates standardized ways to define, register, measure, follow, and supervise R/S and violence.

\section{IMPLEMENTATION IN AUSTRALIA Background}

New South Wales (NSW) Ministry 
of Health was a partner in the National Mental Health Seclusion and Restraint Project (2007-2009) and has continued the commitment to reduce and, where possible, eliminate the use of $\mathrm{R} / \mathrm{S}$ in public mental health services. Until 2011, attention had been on adult mental health inpatient units, and seclusion rates in acute child and adolescent mental health service (CAMHS) units were higher than in adult services.

NSW Ministry of Health has 83 specialist acute CAMHS inpatient beds across nine units in metropolitan and regional cities. Three of these units began admissions between 2012 and 2013 . Unit sizes range from six to 12 beds. The age profile for inpatients across NSW acute CAMHS units from July to December 2013 was approximately $3 \%$ ages 6 to $12,56 \%$ ages 13 to 15 , and $40 \%$ ages 16 to 17 , with an average age of 15 and a median length of stay of 20 days (NSW Ministry of Health, 2014). The units are declared under the NSW Mental Health Act 2007, which states that "any restriction on the liberty of patients and other people with a mental illness or mental disorder and any interference with their rights, dignity and self-respect is to be kept to the minimum necessary in the circumstances" (section 68 [f]).

\section{Process}

In 2011, inspired by the work of child and adolescent mental health colleagues in Massachusetts and the evidence supporting their resource guide (Massachusetts Department of Mental Health, 2008), all units agreed to focus on reducing coercive interventions as a priority through its acute CAMHS inpatient benchmarking/"reflecting on practice" quality improvement program. In 2012, all units began to implement the Six Core Strategies across NSW CAMHS inpatient units, as described by each core strategy below.

Leadership Toward Organizational Change. Reduction of R/S in CAMHS units became a regular item on the agendas of statewide mental health advisory and leadership groups. Project leadership and technical support for units was provided through $\mathrm{MH}$-Children and Young People, a unit of the Mental Health and Drug and Alcohol Office in the NSW Ministry of Health. NSW Ministry of Health's commitment to reduce $R / S$ was affirmed in a revised policy directive issued in 2012 (NSW Ministry of Health, 2012). The Six Core Strategies (Huckshorn, 2006) were integral to the National Mental Health Seclusion and Restraint Project and remain a feature of this policy directive. Service leaders in a new, sixbed regional CAMHS unit decided that the final fit-out would not include a seclusion room.

Using Data to Inform Practice. All units were already submitting 6-month reports on seclusion episodes. CAMHS inpatient units began examining their seclusion data, setting agreed reduction goals at joint 6-month benchmarking forums, and working toward these goals. User-friendly graphs were provided through the NSW Clinical Information Benchmarking Report Engine (CIBRE) tool (NSW Ministry of Health, 2014).

Workforce Development. The MHChildren and Young People unit hosted evidence-informed introductory training for service leaders and more comprehensive workshops for clinicians nominated from all NSW CAMHS inpatient units in March 2012. This Creating Positive Cultures of Care training program was delivered by international consultants and encouraged a culture of strengths-based collaborative care to reduce coercive measures.

Restraint and Seclusion Prevention Tools. Historically, staff training in the management of aggression had focused more on response than prevention. Creating Positive Cultures of Care training helped shift attention to prevention. Nominated clinicians from all CAMHS inpatient units were provided with training in sensory assessment and intervention in May 2012, and occupational therapists (OTs) were provided more specialized training. Follow-up training and supervision for OTs was delivered through a university in Sydney. Sensory tools and equip- ment were distributed to all CAMHS inpatient units. Common NSW Ministry of Health guidelines on the safe and appropriate use of sensory equipment in CAMHS inpatient units are being finalized.

Consumer and Family Involvement. Through the consumer and carer partner organizations (i.e., NSW Consumer Advisory Group-Mental Health Inc. and Mental Health Carers ARAFMI NSW), help was enlisted from young consumers and families. These individuals generously shared their personal stories in Creating Positive Cultures of Care training, and the impact on clinicians was profound. The initiative has benefitted from continued support from state consumer and carer organizations. Services have begun developing local young consumer advisory groups.

Debriefing Techniques. Unit leaders acknowledge that implementation of this strategy has not been as robust as the other five strategies, and the debriefing strategy is on their agenda for the next benchmarking forum.

\section{Outcomes}

Median baseline seclusion rates in NSW acute CAMHS units for July to December 2011 were 20.4 episodes of seclusion per 1,000 occupied bed days (the highest since regular reporting began in 2008), with $15.2 \%$ of hospital stays having an episode of seclusion, which is, again, a particularly high figure. Although progress has not always been smooth, several units have more than halved their seclusion rate and the frequency with which it occurs. Median figures for July to December 2013, the most recent available period, are 2.2 episodes per 1,000 occupied bed days and $3.1 \%$ of stays with an episode of seclusion. Both of these indicators are the lowest since regular collection began in 2008. With these reductions, no significant increase has occurred in the average duration of seclusion. All NSW acute CAMHS inpatient units have a number of activities in progress that are guided by the Six Core Strategies, and they continue to report on 


\section{KEYPOINTS}

LeBel, J.L., Duxbury, J.A., Putkonen, A., Sprague, T., Rae, C., \& Sharpe, J. Multinational Experiences of Reducing and Preventing the Use of Restratin and Seclusion. Journal of Psychosocial Nursing and Mental Health Services, $x x(\mathrm{x}), \mathrm{xx}-\mathrm{xx}$.

1. Restraint and seclusion (R/S) have been used for centuries and continue to be used worldwide.

2. Consensus exists regarding the importance of $R / S$ prevention and reduction and the implementation of methods to prevent and reduce $R / S$.

3. Leadership commitment is essential to begin and sustain treatmentenhancing work of reducing and preventing $\mathrm{R} / \mathrm{S}$.

4. Leaders from a growing number of countries have advanced practice, improved outcomes for patients and staff, and implemented innovations in response to the effort to prevent and reduce R/S use.

Do you agree with this article? Disagree? Have a comment or questions? Send an e-mail to the Journal at jpn@healio.com.

these activities in benchmarking forums.

\section{Next Steps}

NSW Ministry of Health has commenced routine collection and reporting of physical and mechanical restraint in mental health units every 6 months and now publicly reports seclusion rate, average duration, and frequency by facility.

\section{IMPLEMENTATION IN THE UNITED KINGDOM \\ Background}

A new effort has begun in North West (NW) England called REsTRAIN YOURSELF, which is an adaptation of the Six Core Strategies approach. NW England comprises five counties, including Cheshire, Greater Manchester, Merseyside, Lancashire, and Cumbria, with 7 million residents. It is the United Kingdom's third most populated region. All eight of the NW Mental Health Trusts that collectively serve a working age population of approximately 4.3 million individuals have expressed a commitment to participate in the project. Sixteen acute mental health wards in NW England will be included, with eight wards implementing REsTRAIN YOURSELF and an additional eight units acting as comparison wards (i.e., controls). Implementation wards will be recruited based on nomination by the participating trust, with selection based on prespecified criteria. To work effectively, the leaders agreed on criteria for team selection, which includes active service user and carer involvement, clinical leadership, quality improvement teams, and a commitment from the management of aggression trainers to implement the training. A comparison ward in the same trust will be selected based on matching criteria (e.g., restraint rates, acuity, gender mix, staff profile).

The primary aim is to reduce the incidence of harm caused to patients and staff in acute mental health wards through a $40 \%$ reduction in physical restraint and to evaluate any changes in patient safety outcomes.

\section{Process}

REsTRAIN YOURSELF has begun. The process and outcomes described below are the project's planned next steps. The immediate process involves implementing the following components: (a) leadership for a positive safety culture (i.e., leadership walk arounds); (b) root-cause analysis (i.e., debriefing); (c) service user-led initiatives, including advanced directives; (d) service user and staff experience; (e) measurement for improvement; (f) early warning signs/scores; (g) safety climate and physical environment; (h) failure mode and effects analysis; and (i) Plan Do See Act cycles.

The project implementation plan has four key components: (a) training the trainers; (b) implementing training across participating teams; (c) improving collaboration to support learning, sharing, and adoption; and (d) evaluation. Continuous measures will be reviewed monthly, including (a) restraint episode rates, (b) incidence of harms caused by restraint; and (c) levels of ward conflict/climate.

\section{Outcomes}

The research team has adopted an overarching multimethod evaluative design, which will focus on understanding how the program has worked, for whom, and in what circumstances. Principally, the task will be to identify causal mechanisms responsible for generating outcomes and the contexts within which these mechanisms are activated.

The objectives of the evaluation are to (a) prepare for the tailoring and implementation of REsTRAIN YOURSELF; (b) undertake pretests to form a diagnostic and contextual analysis of the current baseline for change; and (c) evaluate the process, outcomes, and sustainability of the practice change using posttests and realist evaluation. A quasiexperimental design has been adopted for the quantitative aspect of the evaluation that will run from Months 7 to 22 of the overall 24-month project. Key outcomes (e.g., restraint rates, staff and patient attitudes) will be assessed prior to implementation (i.e., baseline) and following implementation (i.e., endpoint) on wards implementing REsTRAIN YOURSELF, compared with those on matched wards with no implementation. Several standardized measures, as well as qualitative interviews/focus groups and rapid ethnography, will be used.

\section{IMPLICATIONS AND CONCLUSION}

Statistically significant R/S reduc- 
tions have occurred in the United States and other countries that have implemented the Six Core Strategies to reduce and prevent conflict, violence, and coercion in care (LeBel, 2011; NASMHPD, 2014). More countries are in the process of implementing the Six Core Strategies, including Canada, Ireland, New Zealand, and Japan. Innovations in staffing, workforce development, and alternatives to $\mathrm{R} / \mathrm{S}$ have been developed and widely implemented (LeBel \& Champagne, 2010; NASMHPD, 2014). Many organizations have been able to sustain gains made and advance their reduction/prevention efforts, but some have not (NASMHPD, 2014).

Challenges to $\mathrm{R} / \mathrm{S}$ reduction, prevention, and advancing practice are numerous but not insurmountable. Central to sustaining this work at the local, national, or international level is unwavering leadership commitment and the appropriation of the necessary resources to make and support the change over time. Key tasks for promoting systemic $\mathrm{R} / \mathrm{S}$ reduction efforts include strategic action planning, policy development, and application of lessons learned to other R/S-use settings (Huang, 2011). Catalysts for transformative change include traumainformed care and full consumer inclusion (Huckshorn \& LeBel, 2009). However, all of this work rests on a fundamental platform of values that promote the prevention of conflict, violence, and the use of coercion in human service settings (Huckshorn \& LeBel, 2009; LeBel, in press; NASMHPD, 2014). The Six Core Strategies are an effective, multitask method to reduce and prevent $\mathrm{R} / \mathrm{S}$ and create organizational and practice change in human service settings (NASMHPD, 2014). Transsystem application and implementation of the Six Core Strategies outside of behavioral health is beginning to occur (Huang, 2011; LeBel, in press; NASMHPD, 2014). This application provides an important vehicle by which to maintain gains made and mitigate the potential for a return to past practice, or what some have referred to as $\mathrm{R} / \mathrm{S}$ reduction scotoma, forgetting progress was ever made (LeBel, 2008; Sacks, 2002). Drift from $\mathrm{R} / \mathrm{S}$ reduction is a significant risk without collective advancement across services and countries. In every setting, regardless of location or population served, dedicated leaders are needed to embrace the ultimate goal: to strive to eliminate the use of R/S and treatment violence.

\section{REFERENCES}

Azeem, M.W., Aujla, A., Rammerth, M., Binsfeld, G., \& Jones, R.B. (2011). Effectiveness of Six Core Strategies based on trauma informed care in reducing seclusions and restraints at a child and adolescent psychiatric hospital. Journal of Child and Adolescent Psychiatric Nursing, 24, 11-15. doi:10.1111/ j.1744-6171.2010.00262.x

Barton, S.A., Johnson, M.R., \& Price, L.V. (2009). Achieving restraint-free on an inpatient behavioral health unit. Journal of Psychosocial Nursing and Mental Health Services, 47(1), 34-40.

Foucault, M. (1965). Madness and civilization: A history of insanity in the age of reason. New York, NY: Random House.

Hardy, D.W., \& Patel, M. (2011). Reduce inpatient violence: 6 strategies. Current Psychiatry, 10(5), 80-81.

Huang, L. (2011). Alternatives to seclusion and restraint in behavioral health care. Retrieved from http://www.iacc.hhs.gov/events/2011/ slides_larke_huang_051911.pdf

Huckshorn, K.A. (2006). Re-designing state mental health policy to prevent the use of seclusion and restraint. Administration and Policy in Mental Health, 33, 482-491.

Huckshorn, K.A. (2012). Reducing seclusion and restraint use in mental health settings: A phenomenological study of hospital leader and staff experiences (Unpublished doctoral dissertation). Capella University, Minneapolis, $\mathrm{MN}$.

Huckshorn, K.A., \& LeBel, J.L. (2009). Improving safety in mental health treatment settings: Preventing conflict, violence and the use of seclusion and restraint. In S.S. Sharfstein, F.B. Dickerson, \& J.M. Oldham (Eds.), Textbook of hospital psychiatry (pp. 253-265). Washington, DC: American Psychiatric Publishing.

Human Services Research Institute. (2009). Evaluation report: SAMHSA initiative to reduce and eliminate the use of restraint and seclusion. Cambridge, MA: Author.

Jennings, A. (2007). Blueprint for action: Building trauma-informed mental health service systems. Retrieved from http://www.theannainstitute. org/BFA.pdf

LeBel, J. (2008). Regulatory change: A path- way to eliminating seclusion and restraint or "regulatory scotoma"? Psychiatric Services, 59, 194-196. doi:10.1176/appi.ps.59.2.194

LeBel, J. (2011). The business case for preventing and reducing restraint and seclusion use. Retrieved from http://store.samhsa.gov/shin/ content/SMA11-4632/SMA11-4632.pdf.

LeBel, J., \& Champagne, T. (2010). Integrating sensory and trauma-informed interventions: A Massachusetts state initiative, part 2. Mental Health Special Interest Quarterly, $33(2), 1-4$.

LeBel, J., \& Goldstein, R. (2005). The economic cost of using restraint and the value added by restraint reduction or elimination. Psychiatric Services, 56, 1109-1114.

LeBel, J., Huckshorn, K.A., \& Caldwell, B. (2010). Restraint use in residential programs: Why are best practices ignored? Child Welfare, 89, 169-187.

LeBel, J., Huckshorn, K.A., \& Caldwell, B. (2014). Preventing seclusion and restraint in residential programs. In G.M. Blau, B. Caldwell, \& R.E. Lieberman (Eds.), Residential interventions for children, adolescents and families: A best practice guide (pp. 110-125). New York, NY: Routledge.

LeBel, J., \& Urff, J. (In press). Issue brief: From "safe use" to prevention through traumainformed practices: Seclusion and restraint reform efforts since 1998. Rockville, MD: Center for Mental Health Services, Substance Abuse and Mental Health Services Administration.

Lewis, M., Taylor, K., \& Parks, J. (2009). Crisis prevention management: A program to reduce the use of seclusion and restraint in an inpatient mental health setting. Issues in Mental Health Nursing, 30, 159-164. doi:10.1080/01612840802694171

Massachusetts Department of Mental Health. (2008). Creating positive cultures of care resource guide (2nd ed.). Boston, MA: Author.

Mental Health Act 2007 No 8. Sydney, Australia: North South Wales Government.

National Association of State Mental Health Program Directors. (2014). National executive training institute: A training curriculum for the reduction of seclusion and restraint (12th ed.). Alexandria, VA: Author.

National Registry of Evidenced-Based Programs and Practices. (2012). Six Core Strategies ${ }^{\odot}$ to prevent conflict and violence: Reducing the use of seclusion and restraint. Retrieved from http:// www.nrepp.samhsa.gov/ViewIntervention. aspx?id=278

North South Wales Ministry of Health. (2012). Aggression, seclusion $\mathbb{E}$ restraint in mental health facilities in NSW. Retrieved from http:// www0.health.nsw.gov.au/policies/pd/2012/ pdf/PD2012_035.pdf

North South Wales Ministry of Health. (2014). Clinical information benchmarking report engine (CIBRE) version 10.0 for period ending December 2013. North Sydney, Australia: Author.

Putkonen, A., Kuivalainen, S., Louheranta, O., 
Repo-Tiihonen, E., Ryynänen, O.-P., Kautiainen, H., \& Tiihonen, J. (2013). Clusterrandomized controlled trial of reducing seclusion and restraint in secured care of men with schizophrenia. Psychiatric Services, 64, 850-855. doi:10.1176/appi.ps.201200393

Sacks, O. (2002). Scotoma: Forgetting and neglect in science. In E.B. Hook (Ed.), Prematurity in scientific discovery. Los Angeles, CA: University of California Press.

Wale, J.B., Belkin, G.S., \& Moon, R. (2011). Reducing the use of seclusion and restraint in psychiatric emergency and adult inpatient services: Improving patient-centered care. The Permanente Journal, 15(2), 57-62.

Weiss, E.M., Altimari, D., Blint, D.F., \& Megan, K. (1998, October 11). Deadly restraints: A nationwide pattern of death. The Hartford Courant, 1-16.

Dr. LeBel is Director of System Transformation, Department of Mental Health, Boston, Massachusetts; Dr. Duxbury is Professor, Chair in Mental Health Nursing, School of Health, University of Central Lancashire, Preston, Lancashire, United Kingdom; Dr. Putkonen is Forensic Psychiatrist and Researcher, Niuvanniemi Hospital, University of Eastern Finland, Kuopio, Finland; Ms. Sprague is Associate Director, Clinical Service Development and Quality, Ms. Rae is Senior Nurse Advisor, and Ms. Sharpe is Manager, Clinical Measurement and Benchmarking, Mental Health Drug and Alcohol Office, New South Wales Ministry of Health, Sydney, Australia.

The authors have disclosed no potential conflicts of interests, financial or otherwise.

Address correspondence to Janice L. LeBel, PhD, ABPP, Director of System Transformation, Department of Mental Health, 25 Staniford Street, Boston, MA 02114; e-mail: Janice.Lebel@state. ma.us.

Received: August 1, 2014

Accepted: September 3, 2014

Posted: October 15, 2014

doi:10.3928/02793695-20140915-01 